\title{
Comparative Population Pharmacokinetics of Darunavir in SARS-CoV-2 Patients vs. HIV Patients: The Role of Interleukin-6
}

\author{
Pier Giorgio Cojutti ${ }^{1,2} \cdot$ Angela Londero $^{3} \cdot$ Paola Della Siega $^{3} \cdot$ Filippo Givone $^{3} \cdot$ Martina Fabris $^{4} \cdot$ Jessica Biasizzo $^{4}$. \\ Carlo Tascini $^{3} \cdot$ Federico Pea $^{1,2}$ (1)
}

Published online: 27 August 2020

(c) The Author(s) 2020

\begin{abstract}
Background Darunavir is an anti-HIV protease inhibitor repurposed for SARS-CoV-2 treatment.

Objective The aim of this study was to assess the population pharmacokinetics of darunavir in SARS-CoV-2 patients compared with HIV patients.

Methods Two separate models were created by means of a nonlinear mixed-effect approach. The influence of clinical covariates on each basic model was tested and the association of significant covariates with darunavir parameters was assessed at multivariate regression and classification and regression tree (CART) analyses. Monte Carlo simulation assessed the influence of covariates on the darunavir concentration versus time profile.

Results A one-compartment model well-described darunavir concentrations in both groups. In SARS-CoV-2 patients $(n=30)$, interleukin (IL)-6 and body surface area were covariates associated with darunavir oral clearance (CL/F) and volume of distribution $\left(V_{\mathrm{d}}\right)$, respectively; no covariates were identified in HIV patients $(n=25)$. Darunavir CL/F was significantly lower in SARS-CoV-2 patients compared with HIV patients (4.1 vs. $10.3 \mathrm{~L} / \mathrm{h} ; p<0.001)$. CART analysis found that an IL-6 level of $18 \mathrm{pg} / \mathrm{mL}$ may split the SARS-CoV-2 population in patients with low versus high darunavir CL/F (mean \pm standard deviation $3.47 \pm 1.90$ vs. $8.03 \pm 3.24 \mathrm{~L} / \mathrm{h}$; proportion of reduction in error $=0.46$ ). Median (interquartile range) darunavir $\mathrm{CL} / \mathrm{F}$ was significantly lower in SARS-CoV-2 patients with IL-6 levels $\geq 18 \mathrm{pg} / \mathrm{mL}$ than in SARS-CoV-2 patients with IL-6 levels $<18 \mathrm{pg} / \mathrm{mL}$ or HIV patients $(2.78$ [2.16-4.47] vs. 7.24 [5.88-10.38] vs. 9.75 [8.45-13.79] L/h, respectively; $p<0.0001)$. Increasing IL-6 levels affected darunavir concentration versus time simulated profiles. We hypothesized that increases in IL-6 levels associated with severe SARS-CoV-2 disease may downregulate the cytochrome P450 (CYP) 3A4-mediated metabolism of darunavir.
\end{abstract}

Conclusions This is a proof-of-concept of SARS-CoV-2 disease-drug interactions, and may support the need for optimal dose selection of sensitive CYP3A4 substrates in severe SARS-CoV-2 patients.

Electronic supplementary material The online version of this article (https://doi.org/10.1007/s40262-020-00933-8) contains supplementary material, which is available to authorized users.

Federico Pea

federico.pea@uniud.it

Department of Medicine, University of Udine, Udine, Italy

2 Institute of Clinical Pharmacology, Santa Maria della Misericordia University Hospital of Udine, ASUFC, P.le S. Maria della Misericordia 3, 33100 Udine, Italy

3 Clinic of Infectious Diseases, Santa Maria della Misericordia University Hospital of Udine, ASUFC, Udine, Italy

4 Institute of Clinical Pathology, Santa Maria della Misericordia University Hospital of Udine, ASUFC, Udine, Italy

\section{Introduction}

The spread of the 2019 novel coronavirus (SARS-CoV-2) disease that emerged from China last December has reached pandemic proportions and has become a major global health concern. According to the WHO reports, as at 30 July 2020 more than 16.5 million cases and 655,000 deaths have been reported worldwide [1]. Since the beginning of the European outbreak, Italy has been deeply affected [2] and confirmed cases accounted for $1.5 \%$ of the WHO global prevalence estimate [1].

SARS-CoV-2 may cause illness of increasing severity. The earliest report of SARS-CoV-2 from the Chinese Centers for Disease Control and Prevention noted that among 72,314 cases, $81 \%$ were of a mild nature, with an overall 


\section{Key Points}

The anti-HIV protease inhibitor darunavir is one of the antiviral drugs repurposed for SARS-CoV-2 treatment. We developed and compared population pharmacokinetic models of darunavir in SARS-CoV-2 and HIV patients.

The population pharmacokinetics of darunavir may differ in SARS-CoV-2 patients compared with HIV patients. Interleukin (IL)-6 is the most significant clinical covariate affecting the oral clearance of darunavir in patients with severe disease by downregulating cytochrome P450 (CYP) 3A4 activity.

This may represent a proof-of-concept of SARS-CoV-2 disease-drug interactions, and may support the need for optimal dose selection of any sensitive CYP3A4 substrate in SARS-CoV-2 patients with severe disease.

case fatality rate of $2.3 \%$, whereas $5 \%$ presented with respiratory failure, septic shock, and multiorgan dysfunction, resulting in a fatality rate of $50 \%$ of such cases [1]. Consequently, a three-stage classification system has recently been proposed for characterizing the increasing severity of illness with distinct clinical findings, response to therapy, and clinical outcome: stage I (mild), early infection; stage II (moderate), pulmonary involvement (IIa) without and (IIb) with hypoxia (defined as $\mathrm{PaO}_{2} / \mathrm{FiO}_{2} \leq 300 \mathrm{mmHg}$ ); and stage III (severe), systemic hyperinflammation [3]. Stages I and IIa have been mainly associated with viral proliferation and less severe disease, while stages IIb and III have been associated with overwhelming inflammatory response and cytokine burden, which may precipitate in multiple organ failure, with unfavorable clinical outcome and death $[4,5]$. Although no approved drug treatment currently exists for SARS-CoV-2 disease, hundreds of clinical trials are currently ongoing with the intention of finding an effective treatment, looking at the available therapeutic armamentarium [6]. These trials include various drugs with antiviral activity, immunomodulatory activity, or both [6].

The anti-HIV protease inhibitor darunavir is one of the antiviral drugs repurposed for SARS-CoV-2 treatment, and is currently under investigation in six different clinical trials registered at ClinicalTrials.gov [6]. Three trials included darunavir in the darunavir/cobicistat (DRV/COBI) formulation, and the remaining three trials included darunavir/ritonavir (DRV/RTV) [6]. During the overwhelming outbreak of SARS-CoV-2 disease that occurred in March/April 2020 in Italy, some SARS-CoV-2 patients who were hospitalized at the Clinic of Infectious Diseases of the Udine University
Hospital in the Friuli Venezia Giulia Region were treated with darunavir. These patients underwent routine therapeutic drug monitoring (TDM) during treatment and we noticed that in several cases, plasma concentrations of darunavir were much higher than those usually observed among HIV patients treated with the same drug. Since darunavir is metabolized by cytochrome P450 (CYP) 3A4 in the liver and the gut [7], we hypothesized that SARS-CoV-2 disease might have affected CYP3A4 activity.

The aim of this study was to assess the population pharmacokinetics of darunavir in SARS-CoV-2 patients compared with HIV patients, for identifying any potential covariates that could affect the pharmacokinetics of darunavir in SARS-CoV-2 patients.

\section{Methods}

\subsection{Study Design}

This was a retrospective, single-center, observational study carried out at the Santa Maria della Misericordia University Hospital of Udine, Italy, between 15 March and 15 May 2020. All SARS-CoV-2 patients admitted at the Clinic of Infectious Diseases who were treated with darunavir were included in this study. The standard formulation of darunavir used was DRV/COBI (800/150 mg/day). In the case of an in-hospital shortage of DRV/COBI at the time of admission, the DRV/RTV formulation (800/100 mg/day) was used. Each SARS-CoV-2 patient received only one type of formulation (either DRV/COBI or DRV/RTV) throughout the whole treatment period. HIV patients treated with a fixed-drug combination of DRV/COBI (800/150 mg/day), emtricitabine (200 mg), and tenofovir (10 mg) [DRV/COBI/ FTC/TAF, Symtuza ${ }^{\circledR}$ ] who underwent TDM of darunavir in the same period as the SARS-CoV-2 subjects were included as the comparator group. The rationale for considering this study design as being reliable for population pharmacokinetic modeling is based on the findings of comparative pharmacokinetic studies between DRV/COBI (800/150 mg/day), either as single agents or in a fixed-drug combination, and DRV/RTV (800/100 mg/day). The full bioequivalence of darunavir was shown, in terms of area under the concentration-time curve (AUC) and peak concentration $\left(C_{\max }\right)$, between the two formulations (AUC geometric mean ratio range $0.96-1.02 ; C_{\max }$ geometric mean ratio range $0.97-1.03)[8,9]$, even if the trough concentrations $\left(C_{\min }\right)$ were approximately $30 \%$ lower for DRV/COBI.

All patients routinely underwent TDM of darunavir. SARS-CoV-2 patients underwent TDM assessment at least $48 \mathrm{~h}$ after commencing therapy, while HIV patients were receiving chronic and stable treatment. In both groups, each patient provided two blood samples-the first just before a 
daily dose for assessing $C_{\min }$, and the second $2 \mathrm{~h}$ after oral drug administration for assessing $C_{\max }$. After blood centrifugation and plasma separation, concentrations of darunavir were measured in plasma using liquid chromatography-tandem mass spectrometry (LC-MS/MS) (Shimadzu LC-20 AB Sciex 3200 Qtrap) by means of a commercially available method (MassTox ${ }^{\circledR}$ TDM Series A Anti-HIV drugs, Catalogue No. 92924; Chromsystems GmbH, Munich, Germany). The lower limit of quantification was $6 \mathrm{ng} / \mathrm{mL}$ [10].

This study was approved by the Regional Ethics Committee of Friuli Venezia Giulia Region, Italy. In light of the retrospective nature of the study, written informed consent was waived, according to the institutional policies for studies with a retrospective design.

The following demographic and clinical data were retrieved from patient records: age, sex, weight, height, diagnosis, comorbidities, coadministered drugs, and drug-related adverse events (if any). The following laboratory parameters were collected on the day of the TDM assessment: serum creatinine, serum albumin, alanine aminotransferase (ALT), aspartate aminotransferase (AST), total bilirubin, $\gamma$-glutamyltransferase $(\gamma$-GT), and interleukin (IL)-6. IL-6 was assessed as a biomarker of acute inflammatory progression in SARS-CoV-2 patients [11] and as a biomarker of chronic inflammatory state in HIV patients [12]. The severity of SARS-CoV-2 disease was classified according to the three-stage classification system proposed by Siddiqi and Mehra [3].

\subsection{Population Pharmacokinetic Modeling}

Two comparative population pharmacokinetic models were created, one for SARS-CoV-2 patients and the other for HIV patients. Darunavir plasma concentrations were analyzed by means of nonlinear mixed-effects modeling using the stochastic approximation expectation maximization (SAEM) algorithm implemented within the Monolix software (version 2019R1; Lixofit, Antony, France). The timepoints of darunavir TDM assessment were exactly those related to the start of treatment in SARS-CoV-2. Conversely, six fixed drug doses were virtually added before timepoints of darunavir TDM assessment in HIV patients for simulating steadystate conditions, since those patients had been under chronic treatment for months/years.

A basic model was developed by comparing one- and two-compartment models with first-order oral absorption and elimination. All individual parameters were considered to be log-normally distributed. Exponential random effects were assumed to describe between-subject variability. Correlations between random effects were tested in the variance-covariance matrix, and implemented into the structural model accordingly. Several error models (constant, proportional, or combined) were tested for describing the residual variability. The most appropriate model was selected on the basis of the following criteria: minimum value of both the objective function value (OFV) and the Bayesian information criteria (BIC), adequacy of the goodness-of-fit plots, and minimum value of the relative standard error (RSE) of the estimated pharmacokinetic parameters.

The potential influence of covariates was tested separately on each basic model of the darunavir pharmacokinetics. The tested covariates were age, sex, weight, height, body surface area (BSA), serum albumin, serum creatinine, total bilirubin, ALT, AST, $\gamma$-GT, IL-6, and stage of SARS-CoV-2 disease according to the three-stage classification system of Siddiqi and Mehra [3]. The parameter-covariate relationship was modeled as additive or proportional shifts from the reference category for binary covariates, and the effect of continuous covariates was modeled using a power function. The covariate model was built using a stepwise procedure with forward inclusion and backward elimination. A covariate was retained in each of the two final models if the OFV decreased by at least 3.84 and both the BIC and interpatient variability of the fixed-effect parameters were decreased compared with the corresponding basic model.

\subsection{Model Evaluation}

Evaluation of each final model was based on the following goodness-of-fit plots: observation versus individual and population predictions, usual residual-based plots (individualweighted residuals and population-weighted residuals), and visual predicted check (VPC) plot. The VPC plot depicts the time course of the 10th, 50th, and 90th percentiles of observed data and the corresponding $90 \%$ prediction intervals calculated from 500 Monte Carlo samples. Model performance was also assessed by means of the normalized prediction distribution error (NPDE). The $99 \%$ confidence interval of each parameter was simulated in each final model by performing 1000 nonparametric bootstraps with resampling in the Rsmlx package of $\mathrm{R}$ ( $\mathrm{R}$ speaks Monolix).

\subsection{Monte Carlo Simulation of the Effect of Covariates}

The influence of a covariate eventually retained in the two final models on the plasma concentration versus time profile of darunavir was assessed by means of the Mlxplore package of Monolix. Overall, 1000 Monte Carlo simulations were conducted in each scenario using a 10-day standard daily dose of darunavir $800 \mathrm{mg}$ (simulating either DRV/COBI $800 / 150 \mathrm{mg}$ or DRV/RTV 800/100 mg).

\subsection{Statistical Analysis}

The Kolmogorov-Smirnov test was used for assessing normal or non-normal distribution of patients' data. 
Consequently, descriptive statistical data were presented as mean \pm standard deviation (SD) or median and 25 th -75 th percentiles (interquartile range $[\mathrm{IQR}]$ ). Categorical variables were compared using the Chi square test with Yates's correction, or Fisher's exact test, as appropriate, while continuous variables were compared using the Student's $t$ test or Mann-Whitney test, as appropriate. The strength of a trend between two variables was expressed by means of the Pearson correlation coefficient or Spearman's rank correlation coefficient, as appropriate. Univariate and multivariate linear regression analysis was conducted for assessing the potential association between patient covariates and darunavir pharmacokinetic parameters. A $p$-value $\leq 0.05$ was required for statistical significance. Classification and regression tree (CART) analysis was carried out for assessing whether a covariate threshold could be associated with darunavir pharmacokinetic parameters. The proportion of reduction in error (PRE) was used as goodness-of-fit statistics for CART analysis, and the Kruskal-Wallis test was used to compare continuous data among groups. Bonferroni correction for multiple comparison was applied as appropriate. All statistical analysis and plotting was performed using $\mathrm{R}$ version 3.4.4 (The R Foundation for Statistical Computing, Vienna, Austria).

\section{Results}

Thirty SARS-CoV-2 patients and 25 HIV patients were included in this study. The demographic and clinical characteristics of the two groups are summarized in Table 1. The two groups were similar in terms of sex and distribution of body size metric characteristics. Median age was higher in SARS-CoV-2 patients than in HIV patients (63 vs. 47 years; $p<0.001)$. Levels of ALT, AST, bilirubin, and $\gamma$-GT were significantly higher in SARS-CoV-2 patients compared with HIV patients, and IL-6 levels were highly significantly higher in SARS-CoV-2 patients than in HIV patients (median 31.0 [IQR 10-114.75] vs. 2.0 [IQR 2.0-2.75] pg/ $\mathrm{mL} ; p<0.001)$.

The clinical data of each of the SARS-CoV-2 and HIV patients are summarized in Electronic Supplementary Tables S1 and S2, respectively. Among the SARS-CoV-2 patients (Electronic Supplementary Table S1), 22 of 30 patients $(73.3 \%)$ were treated with DRV/COBI, whereas 8 of $30(26.7 \%)$ received DRV/RTV. Most of the SARS-CoV-2 patients were affected by severe disease (three at stage I, eight at stage IIa, ten at stage IIb, and nine at stage III). Seven patients had no comorbidities $(7 / 30,23.3 \%)$, nine had one comorbidity $(9 / 30,30,0 \%)$ and the remaining 14 had two or more comorbidities $(14 / 30,46.7 \%)$. All patients were co-treated with other drugs (the range of coadministered drugs was 1-8). Only one of the coadministered drugs was a potential perpetrator of CYP3A4-mediated drug-drug interactions (amiodarone was started in one patient 2 days before DRV/COBI).

Among the HIV patients (Electronic Supplementary Table S2), all were treated with DRV/COBI/FTC/TAF $(25 / 25,100 \%)$. Ten patients had no comorbidities $(10 / 25$, $40.0 \%)$, five had one comorbidity $(5 / 25,20,0 \%)$, and the remaining ten patients had two or more comorbidities $(10 / 25,40.0 \%)$. Twenty of 25 patients $(20 / 25,80 \%)$ were cotreated with other drugs (the range of coadministered drugs was 1-8). None of the coadministered drugs was a potential perpetrator of CYP3A4-mediated drug-drug interaction.

\subsection{Comparative Population Pharmacokinetic Analysis of Darunavir}

Both the $C_{\min }$ and $C_{\max }$ of darunavir were significantly higher in SARS-CoV-2 patients than HIV patients (median $C_{\text {min }}$ $4960.2 \mathrm{ng} / \mathrm{mL}$ [IQR 2015.0-7951.2] vs. $1010.0 \mathrm{ng} / \mathrm{mL}$ [IQR 550.0-2112.0], $p<0.001$; median $C_{\max } 9476.2 \mathrm{ng} / \mathrm{mL}$ [IQR 7220.5-14835.4] vs. $6320.0 \mathrm{ng} / \mathrm{mL}$ [IQR 3888.3-7747.5], $p<0.001$ ) [Fig. 1].

In both patient populations, darunavir pharmacokinetic concentrations were best described by a one-compartment model (OFV/BIC for one- vs. two- compartment models: 298.93 vs. 321.74 and 299.59 vs. 337.01 , respectively, in SARS-CoV-2 patients; 181.67 vs. 214.42 and 182.55 vs. 217.96, respectively, in HIV patients). Population pharmacokinetic parameter estimates are reported in Table 2 . In the SARS-CoV-2 final pharmacokinetic model, two covariates were significantly associated with darunavir pharmacokinetic parameters: IL-6 with darunavir oral clearance (CL/F), and BSA with volume of distribution $(\mathrm{Vd})$. On the contrary, no covariate was found to be significantly associated with any of the darunavir pharmacokinetic parameters in the HIV final pharmacokinetic model. Either fixed-effect or randomeffect estimates were accurate in both models (except for interindividual variability in $\mathrm{Vd}$ ). All of the pharmacokinetic parameter estimates were significantly different between the two groups. Noteworthy, darunavir CL/F was approximately 2.5-fold lower (4.1 vs. $10.3 \mathrm{~L} / \mathrm{h} ; p<0.001$ ) and median darunavir exposure was more or less 2.1-fold higher (AUCs of $161,387.0$ vs. $75,727.0 \mathrm{ng} \cdot \mathrm{h} / \mathrm{mL} ; p<0.001)$ in SARS-CoV-2 patients compared with HIV patients.

Linear regression of the observed versus model-predicted individual concentrations showed a very good fit, for both SARS-CoV-2 patients $\left(R^{2}=0.92\right)$ and HIV patients $\left(R^{2}=0.98\right)$ [Electronic Supplementary Fig. S1]. The VPC plot showed a consistent distribution between the observed and predicted concentrations in both populations (Fig. 2). The residuals were normally distributed $(p=0.344$ and $p=0.159$ in the Shapiro-Wilk test for SARS-CoV-2 and HIV patients, respectively), and were symmetric at approximately 
Table 1 Demographics and clinical characteristics of the study groups

\begin{tabular}{|c|c|c|c|}
\hline & SARS-CoV-2-infected patients & HIV-infected patients & $p$-Value \\
\hline \multicolumn{4}{|l|}{ Patient demographics } \\
\hline No. of patients & 30 & 25 & \\
\hline Sex (male/female) & $18 / 12(60 / 40)$ & $18 / 7(72 / 28)$ & 0.404 \\
\hline Age, years & $63(55-70.5)$ & $47(40-51)$ & $<0.001$ \\
\hline Body weight, $\mathrm{kg}$ & $75.0(69.25-81.50)$ & $75.0(66.0-84.0)$ & 0.904 \\
\hline $\mathrm{BSA}, \mathrm{m}^{2}$ & $1.86(1.77-1.96)$ & $1.86(1.75-2.02)$ & 0.689 \\
\hline \multicolumn{4}{|l|}{ Laboratory parameters } \\
\hline Serum creatinine, $\mathrm{mg} / \mathrm{dL}$ & $0.86(0.78-1.05)$ & $0.93(0.84-1.07)$ & 0.424 \\
\hline Bilirubin, mg/dL & $0.46(0.38-0.62)$ & $0.35(0.23-0.48)$ & 0.026 \\
\hline Alanine aminotransferase, UI/L & $40.0(21.0-71.0)$ & $20.0(17.0-29.0)$ & 0.002 \\
\hline Aspartate aminotransferase, UI/L & $36.0(24.0-55.0)$ & $20.0(17.0-22.0)$ & 0.004 \\
\hline$\gamma$-Glutamyltransferase, UI/L & $53.5(35.5-125.2)$ & $22.0(12.0-35.0)$ & 0.035 \\
\hline Interleukin-6, $\mathrm{pg} / \mathrm{mL}$ & $31.0(10-114.75)$ & $2.0(2.0-2.75)$ & $<0.001$ \\
\hline Post-starting treatment time of TDM, days & $3.0(2.0-5.0)$ & - & - \\
\hline \multicolumn{4}{|l|}{ Comedications } \\
\hline No. of drugs per patient & $3.5(2.0-5.75)$ & $4.0(3.0-6.0)$ & 0.151 \\
\hline \multicolumn{4}{|l|}{ ADRs } \\
\hline No. of patients with ADRs & $8(26.7)$ & $1(4.0)$ & 0.031 \\
\hline \multicolumn{4}{|l|}{ Type of ADRs } \\
\hline Abdominal pain/diarrhea & 3 & 1 & 0.617 \\
\hline QT prolongation & 2 & 0 & - \\
\hline Asthenia & 1 & 0 & - \\
\hline Tonic-clonic seizure & 1 & 0 & - \\
\hline Rash & 1 & 0 & - \\
\hline
\end{tabular}

Data are expressed as median (interquartile range) for continuous variables and $n(\%)$ for dichotomous variables

$A D R s$ adverse drug reactions, $B S A$ body surface area, TDM therapeutic drug monitoring

zero $(p=0.156$ and $p=0.219$ in the symmetry test for SARSCoV-2 and HIV patients, respectively).

\subsection{The Prominent Role of Interleukin-6 on Darunavir Pharmacokinetics in SARS-CoV-2 Patients}

IL-6 showed a trend toward a significant relationship with the three-stage classification system of SARS-CoV-2 disease severity (Spearman $\rho=0.34, p=0.07$ ) and was the only clinical variable highly significantly associated with darunavir CL/F at multivariate regression analysis $(p<0.001)$ [Table 3]. The multivariate model accounted for approximately $50 \%$ of the darunavir CL/F variability $\left(R^{2}=0.494\right.$; $p<0.001$ ). CART analysis (Electronic Supplementary Fig. S2) found that an IL-6 level of $18 \mathrm{pg} / \mathrm{mL}$ may adequately split the SARS-CoV-2 population in patients with low versus high darunavir $\mathrm{CL} / \mathrm{F}$ (mean $\pm \mathrm{SD} 3.47 \pm 1.90$ vs. $8.03 \pm 3.24 \mathrm{~L} / \mathrm{h} ; \mathrm{PRE}=0.46)$.

The distribution of darunavir CL/F in SARS-CoV-2 patients with IL-6 levels $\geq 18 \mathrm{pg} / \mathrm{mL}$ compared with those with IL-6 levels $<18 \mathrm{pg} / \mathrm{mL}$, and HIV patients, are depicted in Fig. 3. Median darunavir CL/F was significantly lower in SARS-CoV-2 patients with IL-6 levels $\geq 18 \mathrm{pg} / \mathrm{mL}$ than in those with IL-6 levels $<18 \mathrm{pg} / \mathrm{mL}$, or HIV patients $(2.78$ [IQR 2.16-4.47] vs. 7.24 [IQR 5.88-10.38] vs. 9.75 [IQR 8.45-13.79] L/h, respectively; $p<0.0001)$. Conversely, darunavir CL/F in SARS-CoV-2 patients with IL-6 levels $<18 \mathrm{pg} / \mathrm{mL}$ was not significantly different from that of HIV patients (7.24 [IQR 5.88-10.38] vs. 9.75 [IQR 8.45-13.79] L/h; $p=0.126]$.

Figure 4 depicts the simulated median plasma concentration versus time profiles achievable with a 10-day course of darunavir in HIV and SARS-CoV-2 patients with three different increasing levels of IL-6. Median darunavir $C_{\min }$ and $C_{\max }$ estimates at steady-state were 826 (IQR 239-1824) and 5.425 (IQR 4.229-6.921) ng/mL in HIV patients; 1460 (IQR 550-2920) and 7.560 (IQR 6.147-9.242) ng/mL in SARS-CoV-2 patients with IL-6 levels of $1 \mathrm{pg} / \mathrm{mL} ; 7020$ (IQR 4090-11,380) and 13.418 (IQR 10.468-17.911) ng/ $\mathrm{mL}$ in patients with IL-6 levels of $100 \mathrm{pg} / \mathrm{mL}$; and 14,140 (IQR 9100-21,230) and 20.729 (IQR 15.541-27.778) ng/ $\mathrm{mL}$ in patients with IL-6 levels of $1000 \mathrm{pg} / \mathrm{mL}$. 
Fig. 1 Box-and-whisker plots (5th and 95th percentiles) of darunavir trough and peak plasma concentrations in SARSCoV-2 patients (blue, $n=30$ ) and HIV patients (orange, $n=25)$

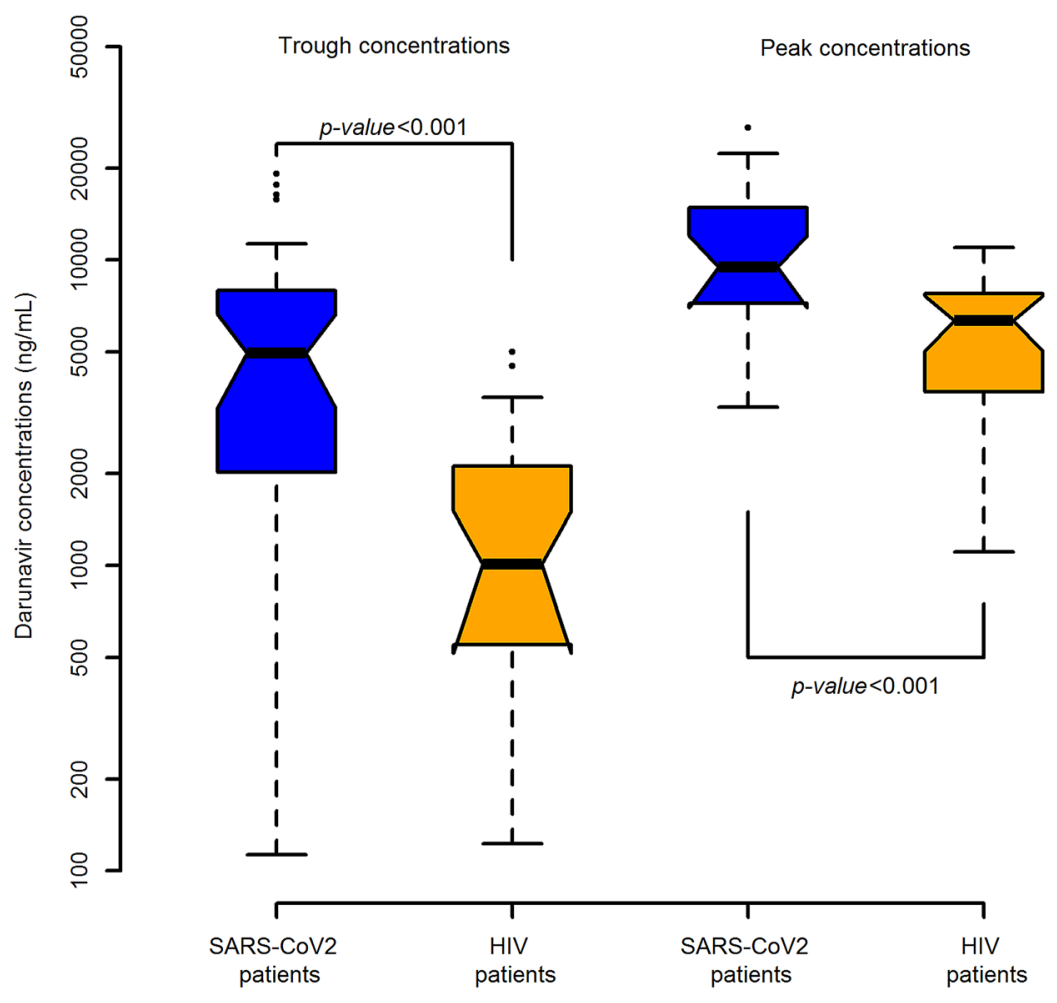

Table 2 Summary of population pharmacokinetic parameters of darunavir in SARS-CoV-2 patients compared with HIV patients

\begin{tabular}{|c|c|c|c|c|c|}
\hline & \multicolumn{2}{|c|}{ SARS-CoV-2 patients } & \multicolumn{2}{|l|}{ HIV patients } & \multirow[t]{2}{*}{$p$-Value } \\
\hline & Value (RSE\%) & Median ( $99 \%$ CI) of bootstrap & Value (RSE\%) & Median ( $99 \%$ CI) of bootstrap & \\
\hline \multicolumn{6}{|l|}{ Fixed effects } \\
\hline$k_{\mathrm{a}}\left(\mathrm{h}^{-1}\right)$ & $0.74(30.6)$ & $0.58(0.55-0.75)$ & $0.58(36.6)$ & $0.59(0.55-0.66)$ & 0.047 \\
\hline $\mathrm{CL} / \mathrm{F}(\mathrm{L} / \mathrm{h})$ & $4.10(10.1)$ & $3.98(3.93-4.11)$ & $10.3(10.4)$ & $10.4(10.2-10.6)$ & $<0.001$ \\
\hline$\beta_{\mathrm{IL6} 6 \mathrm{CL} / \mathrm{F}}$ & $-0.23(24.9)$ & $-0.22(-0.23$ to -0.21$)$ & - & - & - \\
\hline$V_{\mathrm{d}}(\mathrm{L})$ & $88.41(7.8)$ & $77.81(76.13-89.17)$ & $96.9(11.5)$ & $99.4(96.7-102.0)$ & 0.002 \\
\hline$\beta_{\mathrm{BSA}-\mathrm{Vd}}$ & $1.44(35.9)$ & $1.43(1.31-1.71)$ & - & - & - \\
\hline \multicolumn{6}{|c|}{ Between-subject variability } \\
\hline$\omega k_{\mathrm{a}}(\%)$ & $0.82(28.3)$ & $0.97(0.93-1.10)$ & $1.14(24.6)$ & $1.19(1.12-1.25)$ & 0.033 \\
\hline$\omega \mathrm{CL}(\%)$ & $0.53(15.2)$ & $0.53(0.51-0.53)$ & $0.44(19.5)$ & $0.46(0.44-0.48)$ & 0.047 \\
\hline$\omega V_{\mathrm{d}}(\%)$ & $0.15(41.0)$ & $0.13(0.11-0.16)$ & $0.13(61.5)$ & $0.18(0.12-0.20)$ & $<0.001$ \\
\hline \multicolumn{6}{|c|}{ Residual variability } \\
\hline$b$ (proportional) & $0.09(22.3)$ & $0.08(0.07-0.09)$ & $0.155(64.2)$ & $0.17(0.16-0.19)$ & - \\
\hline
\end{tabular}

Results are expressed as estimated parameter value

$\beta_{B S A-V d}$ exponentiation coefficient of BSA on $V_{\mathrm{d}}, \beta_{I L 6-C L / F}$ exponentiation coefficient of IL-6 on CL/F, $C I$ confidence interval, $C L / F$ oral clearance, $k_{a}$ first-order absorption rate constant, $\% R S E$ percentage relative standard error, $V_{d}$ volume of distribution of the central compartment

\section{Discussion}

The present study investigated the population pharmacokinetics of darunavir in SARS-CoV-2 patients compared with
HIV patients. The clinical covariates that may alter darunavir pharmacokinetics in SARS-CoV-2 patients were also assessed, and conceptualization of a disease-drug interaction was formulated. 


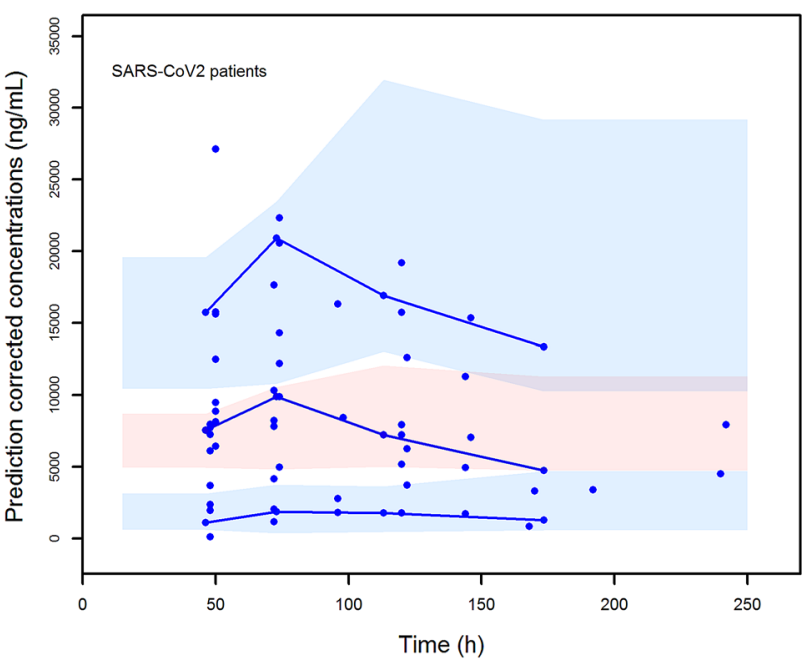

Fig. 2 Prediction-corrected visual predictive check for the population pharmacokinetic models developed in SARS-CoV-2 and HIV patients. The lines represent the median and the 10th and 90th per-

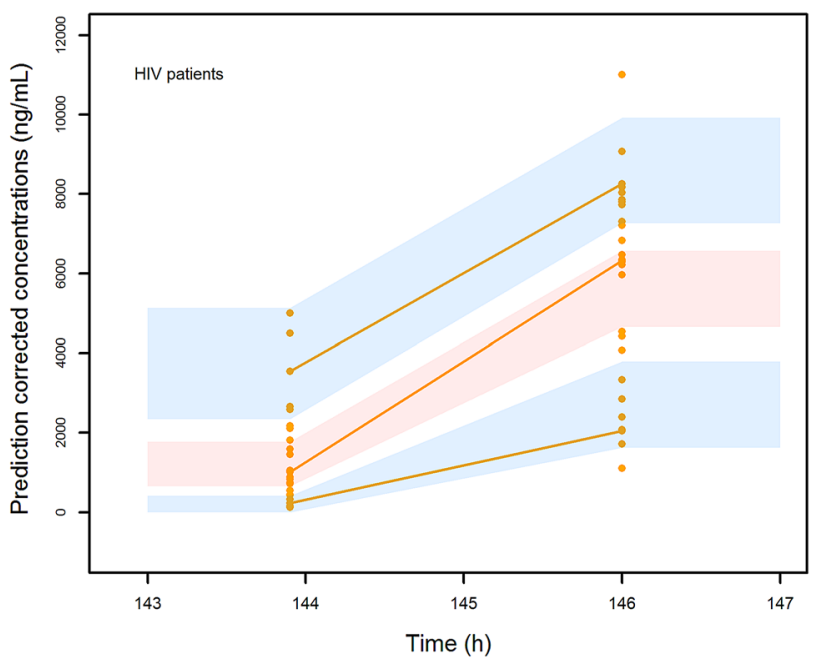

centiles for the observed data. The shaded areas are the prediction interval for the median (red central area) and for the 10th and 90th percentiles (light blue lower and upper areas)

Table 3 Univariate and multivariate analysis of variables potentially associated with darunavir oral clearance in SARS-CoV-2-infected patients $(n=30)$

\begin{tabular}{|c|c|c|c|c|}
\hline \multirow[t]{2}{*}{ Variables } & \multicolumn{2}{|l|}{ Univariate analysis } & \multicolumn{2}{|l|}{ Multivariate analysis } \\
\hline & Unstandardized $\beta$-coefficient & $p$-Value & Unstandardized $\beta$-coefficient & $p$-Value \\
\hline Age, years & $-4.793(-19.078$ to 9.492$)$ & 0.498 & & \\
\hline Body weight, $\mathrm{kg}$ & $8.045(-5.106$ to 21.196$)$ & 0.221 & & \\
\hline $\mathrm{BSA}, \mathrm{m}^{2}$ & $9.237(-16.421$ to 34.895$)$ & 0.467 & & \\
\hline Stage of SARS-CoV-2 progression ${ }^{a}$ & $-6.330(-15.299$ to 2.640$)$ & 0.159 & $-0.668(-7.898$ to 6.561$)$ & 0.851 \\
\hline Serum creatinine, $\mathrm{mg} / \mathrm{dL}$ & $-0.721(-13.672$ to 12.230$)$ & 0.910 & & \\
\hline Total bilirubin, mg/dL & $0.005(-6.673$ to 6.684$)$ & 0.999 & & \\
\hline ALT, UI/L & $0.754(-2.892$ to 4.400$)$ & 0.674 & & \\
\hline AST, UI/L & $1.717(-2.805$ to 6.238$)$ & 0.442 & & \\
\hline$\gamma$-GT, UI/L & $0.549(-2.584$ to 3.681$)$ & 0.721 & & \\
\hline Serum albumin, g/dL & $-1.437(-8.204$ to 5.330$)$ & 0.663 & & \\
\hline IL-6, pg/mL & $-2.464(-3.783$ to -1.145$)$ & 0.001 & $-2.513(-3.795$ to 1.232$)$ & $<0.001$ \\
\hline Hydroxychloroquine & $0.513(-3.577$ to 4.603$)$ & 0.799 & & \\
\hline
\end{tabular}

Results are expressed as slope (confidence interval). Statistical analysis by linear regression on log-transformed independent variables $(Y=$ $\left.\beta_{0}+\beta_{1} \log X+\varepsilon\right)$. Adjusted $R^{2}: 0.494$

Variables significantly associated with darunavir oral clearance at multivariate analysis are in bold

$A L T$ alanine aminotransferase, $A S T$ aspartate aminotransferase, $B S A$ body surface area, $\gamma$ - $G T \gamma$-glutamyltransferase, $I L$ interleukin

${ }^{\mathrm{a}}$ Classified according to the work of Siddiqi and Mehra [3]

The population pharmacokinetic models that we developed showed darunavir CL/F was highly significantly lower in SARS-CoV-2 patients than in HIV patients, and that IL-6 was the only clinical covariate that significantly affected darunavir CL/F in SARS-CoV-2 patients.

The population pharmacokinetic model that we developed in HIV patients receiving DRV/COBI/FTC/TAF was reliable for comparison with SARS-CoV-2 patients as it provided darunavir $\mathrm{CL} / \mathrm{F}$ estimates $(10.3 \mathrm{~L} / \mathrm{h})$ similar to those observed in two recent population pharmacokinetic analysis carried out in HIV patients receiving DRV/RTV. In a recent multicenter, retrospective study assessing the population pharmacokinetics of darunavir among $368 \mathrm{HIV}$ patients treated with DRV/RTV (either in a 800/100 mg once-daily formulation $[n=306]$ or a $600 / 100 \mathrm{mg}$ twice-daily formulation $[n=62]$ ) darunavir CL/F was $9.47 \mathrm{~L} / \mathrm{h}$ [13], and was 
Fig. 3 Scatter plot of the distribution of darunavir $\mathrm{CL} / \mathrm{F}$ in SARS-CoV-2 patients with IL-6 levels $<18 \mathrm{pg} / \mathrm{mL}$ (light blue, $n=10$ ), SARS-CoV-2 patients with IL-6 levels $\geq 18 \mathrm{pg}$ / $\mathrm{mL}$ (purple, $n=20$ ), and HIV patients (orange, $n=25$ ). $C L / F$ oral clearance, $I L$ interleukin
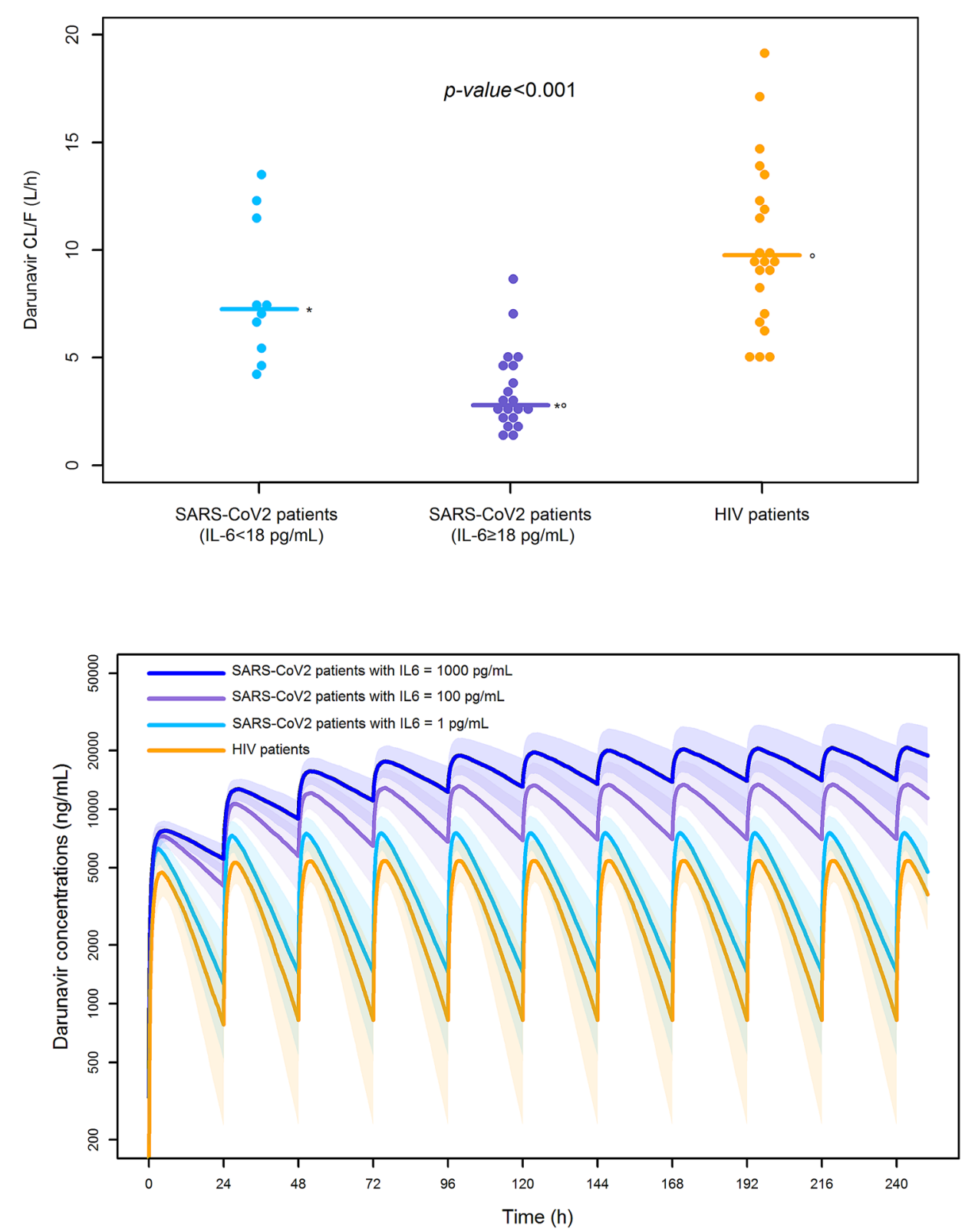

Time (h)
Fig. 4 Simulated median concentration-time profiles of darunavir with a daily dose of $800 \mathrm{mg}$ in HIV patients (orange) and SARS-CoV-2 patients with IL-6 levels of $1 \mathrm{pg} / \mathrm{mL}$ (light blue), $100 \mathrm{pg} /$ $\mathrm{mL}$ (purple), and $1000 \mathrm{pg} / \mathrm{mL}$ (blue). Gray shadings around simulated profiles display the 25th-75th percentiles of concentrations. $I L$ interleukin even higher $(14.6 \mathrm{~L} / \mathrm{h})$ in another population pharmacokinetic study carried out using data from 716 treatment-naïve patients enrolled in a phase III comparative trial of DRT/ RTV 800/100 mg once daily combined with raltegravir in one arm or FTC/TAF in the other arm [14].

To our knowledge, this is the first study assessing darunavir population pharmacokinetics in SARS-CoV-2 patients. We found that darunavir CL/F was approximately 2.5 -fold lower in SARS-CoV-2 patients than in HIV patients, and that IL-6 was the only clinical covariate that significantly affected darunavir CL/F. Darunavir is metabolized by CYP3A4 in the liver and gut [7]. IL-6 is one of the most important proinflammatory cytokines regulating acute-phase protein synthesis in inflammation [15]. Previous studies showed that IL-6 increases associated with infection or other disease states may downregulate CYP3A4-mediated activity [16-19]. Noteworthy, IL-6 burden was shown to play a pivotal role in causing systemic hyperinflammation associated with severe disease states in SARS-CoV-2 patients [20]. Recent evidence from different groups showed a twofold higher lopinavir $C_{\min }$ in SARS-CoV-2 patients [7, 21, 22]. Consequently, some observational studies showed the IL-6 receptor antagonist tocilizumab may be helpful in reducing the fatality rate among patients with severe SARSCoV-2 [20, 23, 24]; a prospective, randomized clinical trial with tocilizumab has also been designed [25]. Most of our patients had severe SARS-CoV-2 disease (overall, 63.3\% were classified as stage IIb and III) [3], and the severity of 
SARS-CoV-2 disease was found to be almost significantly correlated with IL-6 levels.

CART analysis revealed that an IL-6 level $\geq 18 \mathrm{pg} / \mathrm{mL}$ allowed identifying the SARS-CoV-2 patients who had darunavir CL/F most significantly impaired. Conversely, darunavir CL/F in SARS-CoV-2 patients with IL-6 levels below this threshold was almost normal and was similar to that of HIV patients. Interestingly, a physiologically based pharmacokinetic model assessing the impact of IL-6 on sensitive CYP3A4 substrates in virtual rheumatoid arthritis patients may corroborate our hypothesis. In that study, it was estimated that the percentage increase of CYP3A4 victim drug $\mathrm{AUC}_{24}$ in the presence of IL-6 was $20-50 \%$ for simvastatin at IL-6 concentrations of $50 \mathrm{pg} / \mathrm{mL}$, and $30 \%$ for cyclosporine at IL-6 concentrations of $100 \mathrm{pg} / \mathrm{mL}$ [26].

Monte Carlo simulations confirmed that the plasma concentration-time profiles of darunavir achievable with a 10-day course of darunavir may be highly influenced by IL-6 levels in SARS-CoV-2 patients. This may represent a proof-of-concept of SARS-CoV-2 disease-drug interactions, and may support the need for optimal dose selection for darunavir, as well as other sensitive CYP3A4 substrates. The findings could claim dosing adjustments of darunavir in SARS-CoV-2 patients according to their IL-6 concentrations. However, it should not be overlooked that recent studies have shown that the probably of in vivo concentrations of HIV protease inhibitors are insufficient to inhibit SARSCoV-2 [11, 27]. Consequently, it might be hypothesized that the higher exposure observed in the presence of high IL-6 levels could be more clinically helpful in inhibiting SARS-CoV-2.

Prospective studies are urgently needed for promoting effective and well tolerated drug treatments with sensitive CYP3A4 substrates in severe SARS-CoV-2 patients with high IL-6 levels associated with stage IIb and III disease, in agreement with what has already been suggested in a recent viewpoint claiming for action [28].

We recognize that this study has some limitations. Its retrospective nature, the heterogeneity of the different types of darunavir formulations, and the small sample size of both groups must be acknowledged. Although we recognize that a unique population pharmacokinetic model could have been managed, we preferred to create two separate models because we believed that knowing the pharmacokinetic parameter estimates within each single population would have been interesting. The potential influence of CYP3A4mediated drug-drug interactions, OATP1A2 transportermediated hydroxychloroquine interaction, and/or higher levels of AST/ALT on darunavir CL/F, as supposed by other authors [7], could not be completely ruled out; however, it is very unlikely that these factors might have had a major role. Only one of our patients was treated with a known perpetrator of CYP3A4, and neither hydroxychloroquine nor ALT/AST were significantly associated with darunavir $\mathrm{CL} / \mathrm{F}$ at the regression analysis. Conversely, the reliability of the parameter estimates in both population pharmacokinetic models, and the robust significance of IL- 6 as a clinical covariate of darunavir CL/F in SARS-CoV-2 patients, are valuable points of strength.

\section{Conclusion}

Our findings showed that darunavir CL/F may be significantly impaired in SARS-CoV-2 patients, and that IL-6 burden may be the clinical covariate that most significantly affects darunavir $\mathrm{CL} / \mathrm{F}$ in patients with severe disease. Further prospective studies on larger SARS-CoV-2 populations are warranted to confirm our findings. A call for the appropriate application of clinical pharmacological principles in the search for well tolerated and efficacious SARS-CoV-2 treatments is urgently needed [29].

\section{Declarations}

Funding Open access funding provided by Università degli Studi di Udine within the CRUI-CARE Agreement. This research received no external funding.

Conflict of Interest F.P. participated in speaker bureau for Angelini, Basilea Pharmaceutica, Gilead, Hikma, Merck Sharp \& Dohme, Nordic Pharma, Pfizer and Sanofi Aventis, and in advisory board for Angelini, Basilea Pharmaceutica, Correvio, Gilead, Hikma, Merck Sharp \& Dohme, Nordic Pharma, Novartis, Pfizer, Shionogi and Thermo-Fisher. A.L. participated in speaker bureau for Viiv, Gilead, Merck Sharp \& Dohme and Janssen. C.T. participated in speaker bureau and received unconditional grants from Angelini, Biomerieux, Biotest, Gilead, Correvio, Hikma, Merck Sharp \& Dohme, Pfizer, Shionogi, Thermo-Fisher, and Nordic Pharma. All other authors have no conflict of interest.

Ethics Approval The study was approved by the Regional Ethics Committee of Friuli Venezia Giulia Region, Italy.

Consent to participate In light of the retrospective nature of the study, informed written consent was waived, according to the institutional policies for studies with a retrospective design.

Consent for publication In light of the retrospective nature of the study, informed written consent was waived, according to the institutional policies for studies with a retrospective design.

Availability of data and material Not applicable.

Code availability Not applicable.

Authors' contributions PGC and FP conceptualized the study, performed the analysis and drafted the manuscript; AL, PDS and FG acquired and interpreted clinical data; MF and JB performed the laboratory analysis; FP and CT supervised the project and reviewed the entire contents of the manuscript. All authors have read and agree to the published version of the manuscript. 
Open Access This article is licensed under a Creative Commons Attribution-NonCommercial 4.0 International License, which permits any non-commercial use, sharing, adaptation, distribution and reproduction in any medium or format, as long as you give appropriate credit to the original author(s) and the source, provide a link to the Creative Commons licence, and indicate if changes were made. The images or other third party material in this article are included in the article's Creative Commons licence, unless indicated otherwise in a credit line to the material. If material is not included in the article's Creative Commons licence and your intended use is not permitted by statutory regulation or exceeds the permitted use, you will need to obtain permission directly from the copyright holder. To view a copy of this licence, visit http://creativecommons.org/licenses/by-nc/4.0/.

\section{References}

1. WHO. Coronavirus disease (COVID-19) situation reports. 2020 [cited 30 July 2020]. https://www.who.int/emergencies/diseases/ novel-coronavirus-2019/situation-reports. Accessed 31 July 2020.

2. Remuzzi A, Remuzzi G. COVID-19 and Italy: what next? Lancet. 2020;395(10231):1225-8.

3. Siddiqi HK, Mehra MR. COVID-19 illness in native and immunosuppressed states: a clinical-therapeutic staging proposal. J Heart Lung Transplant. 2020;39(5):405-7.

4. Mehta P, McAuley DF, Brown M, Sanchez E, Tattersall RS, Manson JJ, et al. COVID-19: consider cytokine storm syndromes and immunosuppression. Lancet. 2020;395(10229):1033-4.

5. Wu C, Chen X, Cai Y, Xia J, Zhou X, Xu S, et al. Risk factors associated with acute respiratory distress syndrome and death in patients with coronavirus disease 2019 pneumonia in Wuhan, China. JAMA Intern Med. 2020;180(7):1-11. https://doi. org/10.1001/jamainternmed.2020.0994.

6. Lythgoe MP, Middleton P. Ongoing clinical trials for the management of the COVID-19 pandemic. Trends Pharmacol Sci. 2020;41(6):363-82.

7. Baldelli S, Corbellino M, Clementi E, Cattaneo D, Gervasoni C. Lopinavir/ritonavir in COVID-19 patients: maybe yes, but at what dose? J Antimicrob Chemother. 2020. https://doi.org/10.1093/jac/ dkaa190 (Epub 14 May 2020).

8. Kakuda TN, Opsomer M, Timmers M, Iterbeke K, Van De Casteele T, Hillewaert V, et al. Pharmacokinetics of darunavir in fixed-dose combination with cobicistat compared with coadministration of darunavir and ritonavir as single agents in healthy volunteers. J Clin Pharmacol. 2014;54(8):949-57.

9. Navarro J, Curran A. Profile of once-daily darunavir/cobicistat fixed-dose combination for the treatment of HIV/AIDS. HIV AIDS (Auckl). 2016;8:175-82.

10. Chromsystems Instruments \& Chemicals GmbH. MassTox TDM Series A. 2014 [cited 3 Jun 2020]. https://www.chromsyste ms.com/products/therapeutic-drug-monitoring/masstox-tdm-serie s-a-anti-hiv-drugs-92924.html. Accessed 31 July 2020.

11. Cattaneo D, Cattaneo D, Gervasoni C, Corbellino M, Galli M, Riva A, et al. Does lopinavir really inhibit SARS-CoV-2? Pharmacol Res. 2020;158:104898.

12. Borges AH, O'Connor JL, Phillips AN, Ronsholt FF, Pett S, Vjecha MJ, et al. Factors associated with plasma IL-6 levels during HIV infection. J Infect Dis. 2015;212(4):585-95.

13. Daskapan A, Tran QTD, Cattaneo D, Gervasoni C, Resnati C, Stienstra Y, et al. Darunavir population pharmacokinetic model based on HIV outpatient data. Ther Drug Monit. 2019;41(1):59-65.

14. Dickinson L, Gurjar R, Stohr W, Bonora S, Owen A, D'Avolio A, et al. Population pharmacokinetics and pharmacogenetics of ritonavir-boosted darunavir in the presence of raltegravir or tenofovir disoproxil fumarate/emtricitabine in HIV-infected adults and the relationship with virological response: a sub-study of the NEAT001/ANRS143 randomized trial. J Antimicrob Chemother. 2020;75(3):628-39.

15. Renton KW. Cytochrome P450 regulation and drug biotransformation during inflammation and infection. Curr Drug Metab. 2004;5(3):235-43.

16. Aitken AE, Richardson TA, Morgan ET. Regulation of drugmetabolizing enzymes and transporters in inflammation. Annu Rev Pharmacol Toxicol. 2006;46:123-49.

17. Morgan ET. Impact of infectious and inflammatory disease on cytochrome P450-mediated drug metabolism and pharmacokinetics. Clin Pharmacol Ther. 2009;85(4):434-8.

18. Evers R, Dallas S, Dickmann LJ, Fahmi OA, Kenny JR, Kraynov E, et al. Critical review of preclinical approaches to investigate cytochrome $\mathrm{p} 450$-mediated therapeutic protein drug-drug interactions and recommendations for best practices: a white paper. Drug Metab Dispos. 2013;41(9):1598-609.

19. Schmitt C, Kuhn B, Zhang X, Kivitz AJ, Grange S. Diseasedrug-drug interaction involving tocilizumab and simvastatin in patients with rheumatoid arthritis. Clin Pharmacol Ther. 2011;89(5):735-40.

20. Zhang C, Wu Z, Li JW, Zhao H, Wang GQ. Cytokine release syndrome (CRS) in severe COVID-19: interleukin-6 receptor antagonist tocilizumab may be the key to reduce the mortality. Int J Antimicrob Agents. 2020;55(5):105954.

21. Schoergenhofer C, Jilma B, Stimpfl T, Karolyi M, Zoufaly A. Pharmacokinetics of lopinavir and ritonavir in patients hospitalized with coronavirus disease 2019 (COVID-19). Ann Intern Med. 2020. https://doi.org/10.7326/m20-1550 (Epub 12 May 2020).

22. Gregoire M, Le Turnier P, Gaborit BJ, Veyrac G, Lecomte R, Boutoille D, et al. Lopinavir pharmacokinetics in COVID-19 patients. J Antimicrob Chemother. 2020. https://doi.org/10.1093/jac/dkaa1 95 (Epub 22 May 2020).

23. Borku Uysal B, Ikitimur H, Yavuzer S, Ikitimur B, Uysal H, Islamoglu MS, et al. Tocilizumab challenge: a series of cytokine storm therapy experiences in hospitalized Covid-19 pneumonia patients. J Med Virol. 2020. https://doi.org/10.1002/jmv.26111 (Epub 2 Jun 2020).

24. Campochiaro C, Della-Torre E, Cavalli G, De Luca G, Ripa M, Boffini N, et al. Efficacy and safety of tocilizumab in severe COVID-19 patients: a single-centre retrospective cohort study. Eur J Intern Med. 2020;76:43-9.

25. Rilinger J, Kern WV, Duerschmied D, Supady A, Bode C, Staudacher DL, et al. A prospective, randomised, double blind placebo-controlled trial to evaluate the efficacy and safety of tocilizumab in patients with severe COVID-19 pneumonia (TOCCOVID): a structured summary of a study protocol for a randomised controlled trial. Trials. 2020;21(1):470.

26. Machavaram KK, Almond LM, Rostami-Hodjegan A, Gardner I, Jamei M, Tay S, et al. A physiologically based pharmacokinetic modeling approach to predict disease-drug interactions: suppression of CYP3A by IL-6. Clin Pharmacol Ther. 2013;94(2):260-8.

27. Smith PF, Dodds M, Bentley D, Yeo K, Rayner C. Dosing will be a key success factor in repurposing antivirals for COVID-19. Br J Clin Pharmacol. 2020. https://doi.org/10.1111/bcp.14314 (Epub 17 Apr 2020)

28. Peck RW, Weiner D, Cook J, Powell JR. A real-world evidence framework for optimising dosing in all patients with COVID-19. Clin Pharmacol Ther. 2020. https://doi.org/10.1002/cpt.1922 (Epub 23 May 2020).

29. Baker EH, Gnjidic D, Kirkpatrick CMJ, Pirmohamed SM, Wright $\mathrm{DFB}$, Zecharia AY. A call for the appropriate application of clinical pharmacological principles in the search for safe and efficacious COVID-19 (SARS-COV-2) treatments. Br J Clin Pharmacol. 2020. https://doi.org/10.1111/bcp.14416 (Epub 8 Jun 2020). 